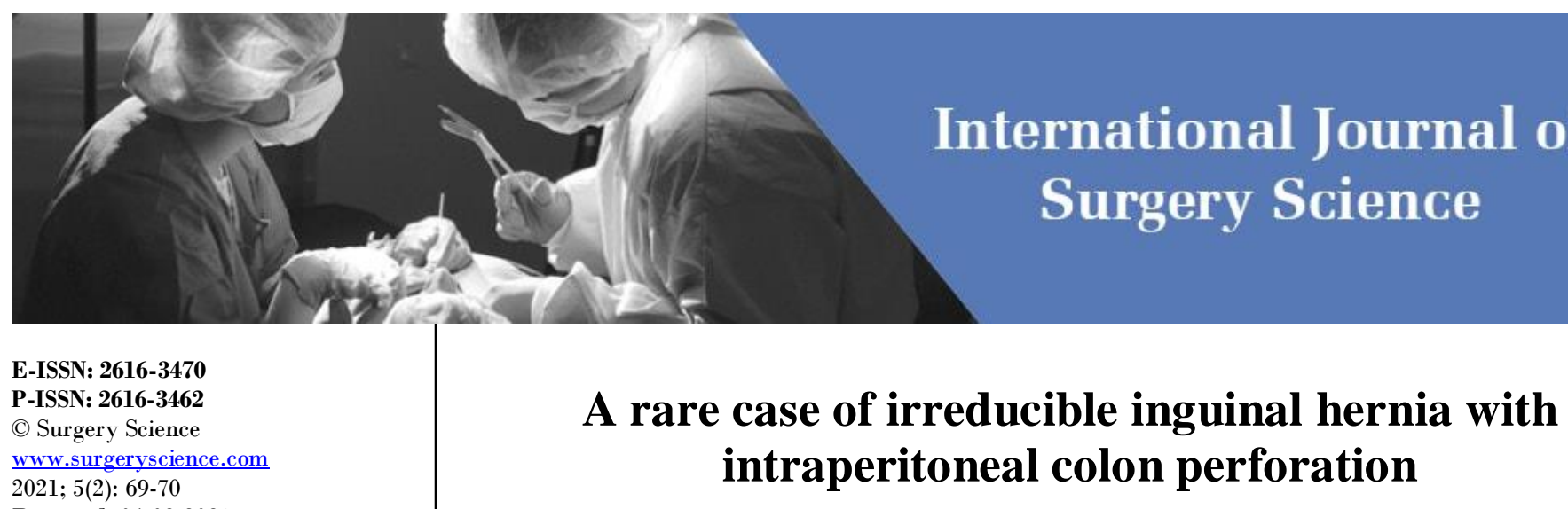

Received: 04-02-2021

Accepted: 06-03-2021

Dr. B Santhi

Professor, Department of General Surgery, Government Kilpauk

Medical College, TN MGR Medical University, Chennai, Tamil Nadu India

Dr. AK Kalpana Devi

Senior Assistant, Professor,

Department of General Surgery,

Government Kilpauk Medical

College, TN MGR Medical

University, Chennai, Tamil Nadu,

India

\section{Dr. Washington}

Assistant Professor, Department of General Surgery, Government

Kilpauk Medical College, TN MGR

Medical University, Chennai,

Tamil Nadu, India

\section{Dr. M Balika}

Post Graduate, Department of General Surgery, Government Kilpauk Medical College, TN MGR Medical University, Chennai, Tamil Nadu, India

\section{Corresponding Author:}

Dr. M Balika

Post Graduate, General Surgery

Govt Kilpauk Medical College

TN MGR Medical University

Chennai, Tamil Nadu, India

\section{Dr. B Santhi, Dr. AK Kalpana Devi, Dr. Washington and Dr. M Balika}

DOI: https://doi.org/10.33545/surgery.2021.v5.i2b.662

\section{Abstract}

The usual incarcerated organs are small bowel and omentum in Inguinal hernias, it is followed by large bowel and other Intra abdominal structures. The cases with sigmoid colon presenting With perforation are rare in the literature. Moreover intra peritoneal Colon perforation is much rarer case, diagnosed clinically and Managed operatively. Herein we would like to present a very unusual Case of irreducible inguinal hernia with intra peritoneal colon Perforation.

Keywords: irreducible, inguinal hernia, intraperitoneal colon perforation

\section{Introduction}

Neglected inguinal hernias may lead to catastrophic results in elderly. Primary goal in elective repair of a reducible hernia is long lasting Closure and prevention of elective hernia recurrence whereas the Goals of emergency repair of a strangulated hernia may be to alleviate Bowel obstruction, debrief devitalized tissue and mitigate the risk of Abdominal catastrophe. Herein we would like to present a very Unusual case of strangulated inguinal hernia.

\section{Case Report}

A $40 \mathrm{yr}$ old male came to us with complaints of swelling in left side of scrotum for past 2 years and Pain in the swelling for the past 1 day which was dragging and continuous. There were no specific aggravating and relieving factors. Pain was not radiating. He had 4 episodes of vomiting which was non bilious, not blood stained.

No history of fever and no history of loose stools or constipation. He had no previous history of surgery and no comorbidities. On general examination, patient was a febrile, tachypneic, Tachycardia present. On inspection, the abdomen was mildly Distended with no visible gastric/ intestinal peristalsis. On palpation, Diffuse tenderness present, no guarding, no rigidity present.

On auscultation, bowel sounds were sluggish. Examination of left Inguino scrotal region showed swelling of left side of scrotum of size $15 \times 15 \mathrm{~cm}$, globular in shape, surface tense, not reducing Spontaneously, no cough impulse. On palpation, the above findings Were confirmed. Warmth and tenderness were present. Not able to get above the swelling. No cough impulse present, swelling not reducible. Examination of opposite inguino scrotal region was within normal limits. Digital rectal examination showed normal sphincter Tone and yellow fecal staining present. Pre operative X Ray abdomen Erect showed no air under diaphragm and no air fluid levels.

Blood investigation showed elevated leukocyte count. Rest of the Investigations were within normal limits. Since hernia was irreducible, patient was tachypneic, had tachycardia, leucocytosis Present, we proceeded with Emergency scrotal exploration. Left Inguino scrotal incision was made and deepened in layers. Hernial sac Opened and found to have omentum as content and fecal matter and Fluid. Hence midline laparotomy done. On exploration, Sigmoid perforation of size $1 \times 1 \mathrm{~cm}$ seen with intact posterior wall. Primary closure was done. Thorough wash given and drains kept. 


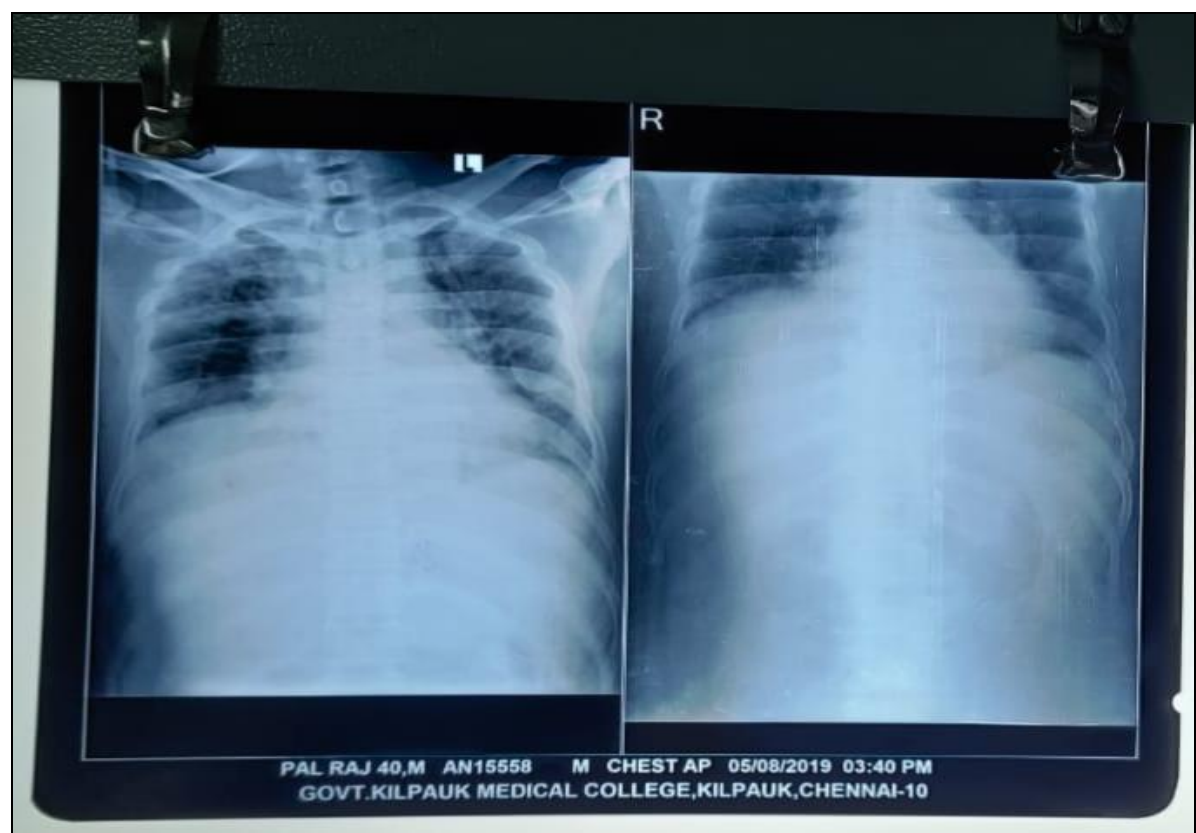

Fig 1: Chest $X$ Ray and $X$ ray abdomen erect

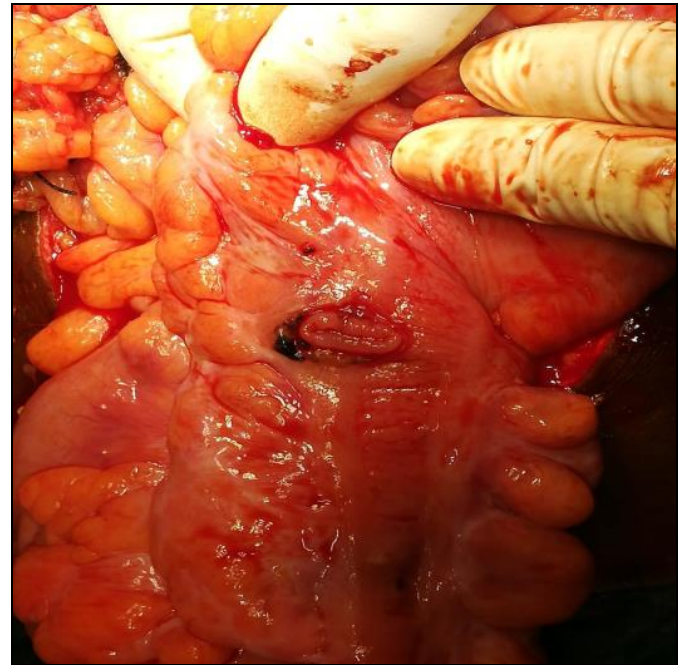

Fig 2: Sigmoid Perforation

Post operatively, vitals were stable. But after 6 hours, Patient became irritable and restless. He started to develop tachycardia and tachypnea. He started to develop crepts in both lungs and his saturation began to fall. Hence he was intubated. He had high grade fever on and off, relieved by medication.

\section{Discussion}

No any such case of complicated inguinal hernia had been reported till now where there is perforation of intra peritoneal part of intestine but no air under diaphragm in X-Ray abdomen erect. It is Diagnosed clinically and managed operatively.

Indirect hernia strangulate more commonly than direct hernia because of narrow neck and adhesion of the contents. Obstructed or strangulated inguinal hernia, perforation does not occur in intra peritoneal part of intestine. Interestingly cases with sigmoid Carcinoma in the hernial sac were reported more frequently.

Incarcerated tumor containing segments may present with Perforation from the tumoral region.

\section{References}

1. Blunting D, Harshen R, Ravichandra $\mathrm{M}$ et al. Unusual Diagnoses presenting as incarcerated inguinal hernia: a case report and review of the literature

2. Bali C, Tsironis A, Zikos N, et al. An unusual case of the strangulated inguinal hernia containing the sigmoid colon.

3. Kulasekaran S, Fernando M, Fraser-Jones B et al. Perforated sigmoid colon carcinoma within a left inguinal hernia with associated necrotising infection 